\title{
PET and PET/CT: A Clinical Guide
}

\author{
C.W. Lin and A. Alavi
}

New York, NY: Thieme, 2009, 297 pages, $\$ 79.95$

PET and PET/CT have been increasingly used as effective imaging modalities for the management of patients with cancer, neurologic diseases, and cardiovascular diseases since the first edition of this concise book was published in 2005. The goal of the first edition was to provide essential information and scientific literature about PET to busy trainees and practitioners in medical imaging fields, in addition to providing an overview of the clinical applications of ${ }^{18} \mathrm{~F}$-FDG PET and PET/CT. This second edition has been updated with more than 500 high-quality PET/CT images, including color images. New chapters related to ${ }^{18} \mathrm{~F}-\mathrm{FDG}$ biology, pediatric PET/CT, and PET/CT in radiation therapy, as well as inflammatory and infectious disorders, have been added. Special emphasis has been placed on the correlation of PET and CT findings.

This practical book is organized into 4 sections with 30 chapters. The first section, "Basic Science," has 3 chapters related to physics, radiochemistry, and biology. The second section, "Clinical Basics," has 6 chapters on patient preparation, normal variants, benign findings, interpretation of ${ }^{18} \mathrm{~F}-\mathrm{FDG}$ PET/CT findings, and clinical indications for ${ }^{18}$ F-FDG PET. The third section, "Oncologic Applications," has 16 chapters discussing various tumors and also covers therapeutic responses. The fourth section, "Nononcological
Applications," has 5 chapters on pediatric PET/CT, PET/CT in radiation therapy planning and in infection and inflammation, and neurologic and cardiac applications.

Each chapter on oncologic applications describes the role of PET and PET/CT in the management of specific diseases, providing succinct descriptions of indications and comparisons with other imaging modalities. Pearls and pitfalls emphasize critical concepts. The outstanding images are clear and illustrative, and the tables are informative. The references are updated, and the index is convenient.

I am sure that this new edition will continuously serve as a valuable reference source of authoritative information on PET and PET/CT. This small and affordable book should be useful to both trainees and practitioners who daily are involved with imaging studies. I highly recommend this compact book to them for better interpretation of PET or $\mathrm{PET} / \mathrm{CT}$ and for sharpening their clinical consultation skills.

\section{E. Edmund Kim}

M.D. Anderson Cancer Center

1515 Holcombe Blvd., Unit 1264

Houston, TX 77030

E-mail:ekim@di.mdacc.tmc.edu

COPYRIGHT (c) 2010 by the Society of Nuclear Medicine, Inc.

DOI: 10.2967/jnumed.109.070276 